\title{
BMJ Open Protocol for a prospective observational study on the association of variables obtained by contrast-enhanced ultrasonography and sepsis-associated acute kidney injury
}

\author{
Ning Liu, ${ }^{1}$ Zhongheng Zhang, ${ }^{\oplus}$ Yucai Hong, ${ }^{1}$ Bing Li, ${ }^{1}$ Huabo Cai, ${ }^{1}$ Hui Zhao, ${ }^{1}$ \\ Junru Dai, ${ }^{1}$ Lian Liu, ${ }^{1}$ Xin Qian, ${ }^{1}$ Qicheng Jin ${ }^{2}$
}

To cite: Liu N, Zhang Z, Hong Y, et al. Protocol for a prospective observational study on the association of variables obtained by contrastenhanced ultrasonography and sepsis-associated acute kidney injury. BMJ Open 2019;9:e023981. doi:10.1136/ bmjopen-2018-023981

\section{- Prepublication history for} this paper is available online. To view these files, please visit the journal online (http://dx.doi org/10.1136/bmjopen-2018023981).

Received 09 May 2018 Revised 05 March 2019 Accepted 08 July 2019

Check for updates

(c) Author(s) (or their employer(s)) 2019. Re-use permitted under CC BY-NC. No commercial re-use. See rights and permissions. Published by BMJ.

${ }^{1}$ Department of Emergency Medicine, Sir Run Run Shaw Hospital, Zhejiang University School of Medicine, Hangzhou, China

${ }^{2}$ Department of Ultrasound Medicine, Sir Run Run Shaw Hospital, Zhejiang University School of Medicine, Hangzhou, China

Correspondence to Dr Zhongheng Zhang; zh_zhang1984@zju.edu.cn

\section{ABSTRACT}

Introduction Sepsis commonly results in acute kidney injury (AKI), whereas about $50 \%$ of AKI cases are due to sepsis. Sepsis-associated acute kidney injury (SA-AKI) increases morbidity and mortality especially among critically ill patients. This study aims to monitor renal microcirculation perfusion during sepsis using contrastenhanced ultrasonography (CEUS), and to explore whether CEUS is useful for predicting the development of SA-AKI. Methods and analysis This prospective observational study will enrol patients who were diagnosed with sepsis-3 definition. The total of septic or septic shock patients were stratified into AKI (including stages 1, 2 and 3) and non-AKI groups according to Kidney Disease Improving Global Outcomes criteria on days 0, 1, 2 and 7 after admission to the emergency intensive care unit, meanwhile, the CEUS technique will be performed to monitor renal microcirculation perfusion. A multivariable model including all CEUS variables were expected to create for predicting the development of AKI during sepsis. Ultrasonography results, demographic information, therapeutic interventions, survival outcomes, laboratory and other clinical datas will also be collected for further analysis.

Ethics and dissemination The study protocol was approved on 2 August 2017 by the Ethics Committee of Sir Run Run Shaw Hospital (Zhejiang University Medical College) (approval number: 2016C91401). The results will be published in a peer-reviewed journal and shared with the worldwide medical community within 2 years after the start of the recruitment.

Trial registration number ISRCTN14728986

\section{INTRODUCTION}

Sepsis is a syndrome that involves an over-activated systemic inflammatory response to infection. This condition can lead to multiple organ dysfunction, with the kidneys being a commonly involved organ. ${ }^{2}$ Acute kidney injury (AKI) is characterised by the loss of kidney function with an increase in serum creatinine and/or a decrease in urinary

\section{Strengths and limitations of this study}

- In this study, we expected to monitor changes of the renal microcirculation including cortex and medullary by contrast-enhanced ultrasonography (CEUS) among patients with sepsis in their first 7 days and try to certify the possibility of redistribution of intrarenal microcirculatory blood flow.

- The CEUS is an uncomplicated and non-nephrotoxic procedure with the rare occurrence of serious side effects and anaphylactic reactions, which should be especially considered in the intensive care unit.

- Limited patients enrolled in the group which will make the available datas inconclusive.

output. ${ }^{3}$ Sepsis is one of the most common causes of AKI, accounting for $50 \%$ of all cases, and up to $60 \%$ of patients with sepsis have AKI. ${ }^{45}$ Sepsis-associated acute kidney injury (SA-AKI) is defined by the simultaneous presence of the recent sepsis-3 consensus criteria for sepsis ${ }^{1}$ and the Kidney Disease Improving Global Outcomes (KDIGO) consensus criteria for AKI. ${ }^{6}$

Current studies indicated that SA-AKI and chronic kidney disease are closely related rather than independent conditions, and even a mild or short-term of SA-AKI have a tendency to develop into chronic and end-stage kidney disease in the near future. ${ }^{78}$ Thus, early prediction and recognition of SA-AKI are important steps in the early and effective initiation of proper therapy, which can prevent further complications and multiple organ dysfunction. Former researches regarding the pathogenesis of SA-AKI mainly focused on the renal macrocirculation resulting from global renal ischaemia, cellular damage and acute tubular necrosis. ${ }^{9-11}$ However, recent advances have enhanced our knowledge of 
the pathobiology of SA-AKI. ${ }^{12-14}$ In SA-AKI, microvascular dysfunction is characterised by a wide heterogeneity in blood fow distribution across the renal tissues and the same microcirculatory derangements have been detected in the renal capillaries. ${ }^{15}$ Therefore, early detection of renal microcirculation dysfunction during and after sepsis may facilitate subsequent treatment and prognostic prediction.

Contrast-enhanced ultrasonography (CEUS) which is a relatively novel modality with microbubble-based contrast agents have provided real-time anatomic and functional information in the study of microvascular fow in patients with SA-AKI without ionising radiation. ${ }^{16}$ Currently, CEUS has become widely used in the emergency department for evaluating cases of abdominal blunt trauma ${ }^{17}$; however, the use of CEUS in SA-AK cases is limited and it remains unclear whether it can provide useful information for predicting the development.

Therefore, as a primary objective, the present study aims to use CEUS at an emergency intensive care unit (EICU) to monitor renal microcirculation perfusion (including cortex and medullary) in the first 7 days of being diagnosed with sepsis. Then, we expected to create a multivariable model including all CEUS parameters to predict the development of SA-AKI.

\section{METHODS AND ANALYSIS \\ Study design}

This prospective observational study will enrol patients with sepsis and septic shock who are treated at an interdisciplinary EICU (Sir Run Run Shaw Hospital, Zhejiang University) between 11 August 2017 and 30 September 2019. All conscious patients will be educated regarding the study's protocol and purposes. If patients were diagnosed with septic shock or even were ventilated and sedated, informed consent will be written by their family members, instead.

\section{Cohort descriptions and definitions}

We plan to recruit 200 patients. Patients with sepsis and septic shock will be screened for eligibility if they are $>18$ years old. The exclusion criteria are (1) an expected stay of $<24$ hours, (2) the presence of end-stage kidney disease or long-term haemodialysis, (3) critically ill patients who have started renal replacement therapy caused by SA-AKI before EICU admission, (4) a history of kidney transplantation and (5) urinary tract obstruction.

Sepsis is defined as life-threatening organ dysfunction caused by a dysregulated host response to infection. For clinical operationalisation, organ dysfunction can be represented by an acute change of $\geq 2$ points in the total Sequential Organ Failure Assessment score. Septic shock is defined as sepsis with persistent hypotension requiring vasopressors to maintain a mean arterial pressure of $\geq 65 \mathrm{~mm} \mathrm{Hg}$ and serum lactate levels of $>2 \mathrm{mmol} / \mathrm{L}$ $(18 \mathrm{mg} / \mathrm{dL})$ despite adequate resuscitation volume (box 1). ${ }^{1}$
Box 1 Sepsis-3 definitions and quick SOFA (qSOFA) criteria

Sepsis-3 definitions

Sepsis-life-threatening organ dysfunction caused by a dysregulated host response to infection.

Septic shock-sepsis with a requirement for vasoactive therapy to maintain mean arterial pressure $\geq 65 \mathrm{~mm} \mathrm{Hg}$ and lactate elevation to

$>2 \mathrm{mmol} / \mathrm{L}$ despite adequate volume resuscitation.

qSOFA criteria

- Respiratory rate $\geq 22$ breaths/min.

- Altered mentation.

- Systolic blood pressure $\leq 100 \mathrm{~mm} \mathrm{Hg}$.

qSOFA, quick SOFA; SOFA, sequential (sepsis-related) organ failure assessment.

In order to identify patients with SA-AKI as early as possible, we could apply the KDIGO guidelines to our subjects, which stratifies SA-AKI in three different stages according to changes in creatinine and urine output (table 1$).{ }^{6}$ Baseline creatinine value $(\mu \mathrm{mol} / \mathrm{L})$ was either registered from 6 months previous clinical files or estimated, when data were not available from clinical records, by solving the modification of diet in renal disease equation assuming a glomerular filtration rate of $75 \mathrm{~mL} / \mathrm{min} / 1.73 \mathrm{~m}^{2} .{ }^{18} 19$

\section{Patient and public involvement}

No patients were involved in developing plans for design or implementation of the study, nor were they involved in setting the research question or the outcome measures. No patients were requested to advise on interpretation or writing up of results. There are no plans to disseminate the results of the research to study participants or the relevant patient community.

\section{Data collection}

Demographic information and chronic disease history will be collected as baseline characteristics during the hospitalisation. The Acute Physiology and Chronic Health Evaluation II score will be used to assess the severity of the disease within 24 hours after EICU admission. Source of infection, use of mechanical ventilation before/after AKI development and therapeutic interventions (the volume and kind of fluid resuscitation, vasopressor therapy, antimicrobial therapy, renal replacement therapy) will be also collected. Each patient will be followed for at least seven consecutive days using routine haematology and biochemistry testing for complete blood count, haemoglobin, arterial blood gas, arteriovenous oxygen difference, probrain natriuretic peptide, plasma protein, $\mathrm{C}$ reactive protein, serum creatinine $(\mathrm{SCr})$ and estimated glomerular filtration rate. The frequency of laboratory tests in patients with stable vital signs is once a day; however, for septic shock patients, laboratory tests may be performed every 8 hours.

Work sheets will also be maintained to record the patients' vital signs (temperature, heart rate, respiration, blood pressure, mean arterial pressure, central venous 


\begin{tabular}{lll}
\hline Table 1 & Staging of sepsis-associated acute kidney injury (SA-AKI) & \\
\hline Stage & Serum creatinine & Urine output \\
\hline 1 & $1.5-1.9$ times baseline OR $\geq 0.3 \mathrm{mg} / \mathrm{dL}(\geq 26.5 \mathrm{mmol} / \mathrm{L})$ increase & $<0.5 \mathrm{~mL} / \mathrm{kg} / \mathrm{hour} \mathrm{for} 6-12 \mathrm{hours}$ \\
2 & $2.0-2.9$ times baseline & $<0.5 \mathrm{~mL} / \mathrm{kg} / \mathrm{hour} \mathrm{for} \geq 12 \mathrm{hours}$ \\
3 & 3.0 times baseline OR increase in serum creatinine to $\geq 4.0 \mathrm{mg} / \mathrm{dL}$ & $<0.3 \mathrm{~mL} / \mathrm{kg} / \mathrm{hour} \mathrm{for} \geq 24 \mathrm{hours} \mathrm{OR}$ \\
& $(\geq 353.6 \mathrm{mmol} / \mathrm{L})$ OR initiation of renal replacement therapy OR, in patients & anuria for $\geq 12 \mathrm{hours}$ \\
& $<18$ years, decrease in eGFR to $<35 \mathrm{~mL} / \mathrm{min} / 1.73 \mathrm{~m}^{2}$ & \\
\hline
\end{tabular}

eGFR, estimated glomerular filtration rate.

pressure, $\mathrm{SpO}_{2}$ ), neurological signs (Glasgow coma score), medication, intake and output. The dosage and frequency will be recorded for vasoactive drugs in cases of shock. Conventional B-mode ultrasonography and CEUS scans will be performed on days 0, 1, 2 and 7 after EICU admission. For septic shock patients, we performed CEUS before fluid resuscitation, and then we would re-evaluate renal microvascular alterations by the use of CEUS with resuscitation targeted at normalisation of blood pressure (mean arterial pressure of CE). All ultrasonography examinations were performed by the collaboration of experienced EICU physicians and ultrasonologist. Perfusion time intensity curve analysis ${ }^{20}$ was performed using the integrated computer workstation (LOGIQ E9, GE). Clinical and biochemical data were retrieved from a clinical database. Data collection was permitted by the ethical review committee of the Sir Run Run Shaw Hospital (Zhejiang University Medical College). Survival outcomes in the EICU, at 1 month, at 3 months, at 1 year and at 2 years after discharge will be determined using outpatient visits or telephone interviews.

\section{Conventional B-mode ultrasonography}

Conventional B-mode ultrasonography scans will be performed using a 3.5-5 MHz convex probe (Mindray M9, China) to evaluate the size, morphology and macrovascular supply of the kidneys, which will be consistently imaged in the longitudinal plane. Colour Doppler flow imaging will be used to evaluate the peak systolic velocity, resistance index and end-diastolic velocities of the segmental artery, interlobar artery and arcuate artery. Cardiac and lung functions will be assessed using bedside echocardiography and lung ultrasonography, ${ }^{22}$ with data including the left ventricular ejection fraction, E-point to septal separation and the respiratory variation of the inferior vena cava diameter.

\section{Performance of CEUS}

The CEUS will be performed immediately after the longaxis view of the kidney has been obtained using conventional B-mode ultrasonography. SonoVue is a commercial ultrasound contrast agent (Bracco, Milan, Italy) that will be administered intravascularly with a bolus of $1-2.4 \mathrm{~mL}$ based on the patient's weight, height and age. The bolus will then be followed by a $10 \mathrm{~mL}$ injection of physiological saline via a peripheral antecubital vein. The timer and imaging recorder will be activated simultaneously with the contrast agent injection, and the procedure will last for a total of 4-6min. A lower mechanical index will be applied, although the mechanical parameters will be standardised for all patients. We will manually define three circular regions of interest (diameters of $1.5-2.0 \mathrm{~cm}$ ) at the renal cortex and medulla, which are at the same approximate location, to analyse renal microcirculation perfusion. Care will be taken to avoid nearby vessels (figure 1). A second contrast agent injection will be performed at an interval of $\sim 15 \mathrm{~min}$ if necessary. Images and video clips from the CEUS will be digitally stored for subsequent quantitative analysis.

The time-intensity curves will be created using software to analyse blood volume and velocity based on the following parameters: baseline intensity (intensity before the contrast agent arrives in the microcirculation), arrival time (the time when the contrast agent appears), timeto-peak (the time to the maximum contrast intensity), peak intensity (the maximum contrast intensity value), ascending slope, descending time/2, descending slope and area under the curve (figure 2). ${ }^{23}$

\section{Statistical analysis}

All statistical analyses will be performed using SPSS software V.17.0 and MedCalc software. Differences will be considered statistically significant at two-tailed $p$ values of $<0.05$. Categorical data will be compared using the $\chi^{2}$ test. Continuous data will be reported as mean $\pm \mathrm{SD}$ and compared using Student's t-test. ${ }^{24}$ Variables that are

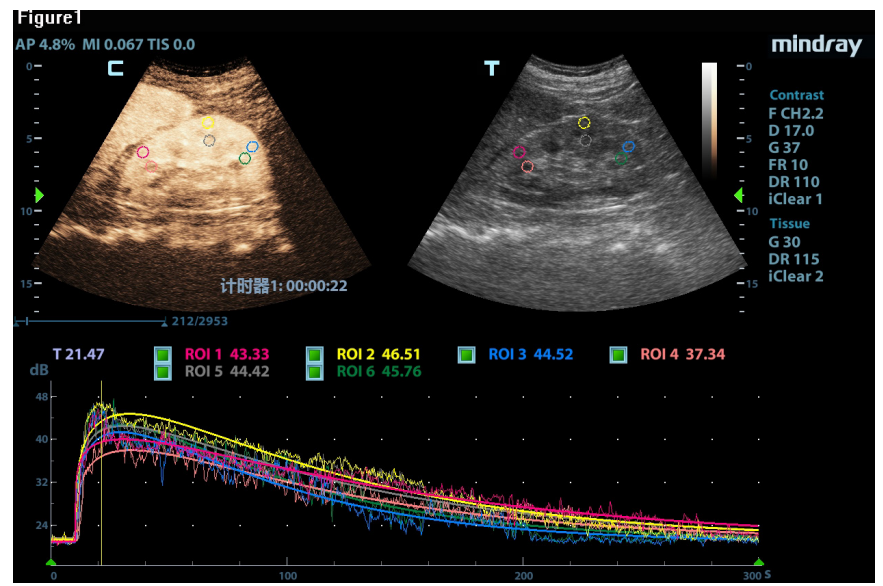

Figure 1 A color-coded map with circular regions of interest (ROIs) at the renal cortex and medulla. 


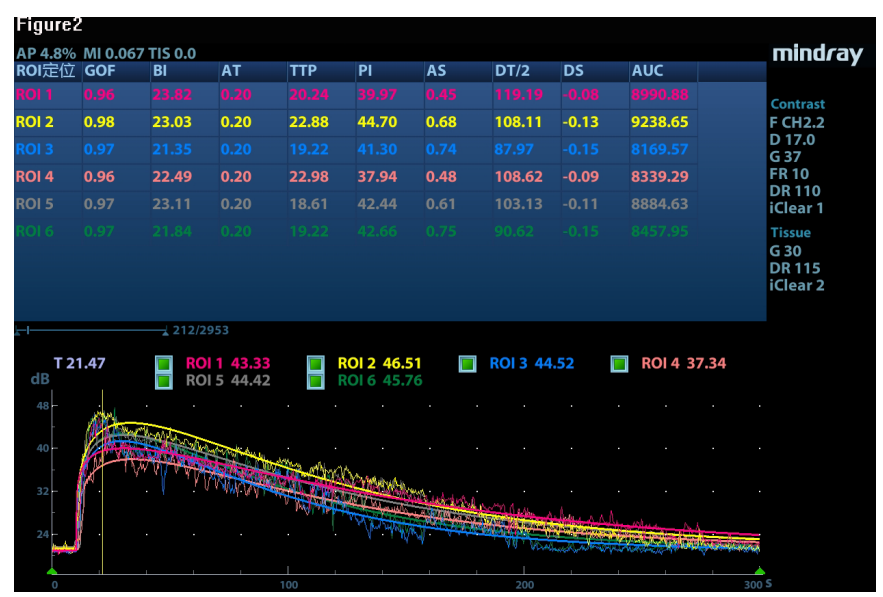

Figure 2 A color-coded graph comparing the quantitative contrast-enhanced ultrasound parameters between the groups with and without acute kidney injury. AS,ascending slope; AT, arrival time; AUC, area under the curve; BI, baselineintensity; DS, descending slope; DT/2, descending time/2; GOF, goodness of fit;PI, peak intensity; TTP, time-topeak.

significantly different between AKI and non-AKI groups in the univariate analyses will be included in multivariate logistic regression analysis. ${ }^{25}$ All CEUS parameters that are statistically significant in univariate analysis will be included in a multivariable regression model to establish a combined score for the predictionof SA-AKI. ${ }^{26} \mathrm{~A}$ receiver operating characteristic curve will also be created to examine the diagnostic performance of CEUS (a multivariable model including all CEUS parameters) to predict AKI. Other diagnostic statistics such as the sensitivity, specificity, accuracy, positive predictive and negative predictive values will be reported for the model.

\section{DISCUSSION}

SA-AKI is common among critically ill patients and is an important cause of ICU admission with significant morbidity and mortality. The incidence of SA-AKI in the ICU is $\sim 55 \%-60 \%$ and the mortality rate is $\sim 27 \%{ }^{27}$ Volume resuscitation, vasopressor therapy, antimicrobial therapy and renal replacement therapy remain the mainstays of the management for SA-AKI in the ICU. ${ }^{28}$ Although SA-AKI is manageable, progression to chronic renal failure can lead to tremendous psychological and economic burdens on the patient. Thus, a better understanding of the pathophysiology of SA-AKI is essential in order to improve the prognosis, disease management and long-term follow-up.

The underlying pathophysiology of SA-AKI is complex and multifactorial. Evidences suggested that the global renal ischaemia, cellular damage and acute tubular necrosis $^{9-11}$ were once regarded as the three main causes leading to the change of the renal macrocirculation in SA-AKI. At present, a growing body of experimental and clinical evidence now shows that, at least in the early phase of SA-AKI, renal blood flow is normal or even increased. ${ }^{14} 29$
Some new study demonstrated that, in the first 48 hours, pathophysiological alterations of SA-AKI may be functional rather than structural in nature, ${ }^{30}$ and potentially reversible. ${ }^{31}$ Therefore, it is crucially important to identify the SA-AKI as early as possible within 48 hours.

To the best of our knowledge, the levels of serum creatinine and blood urea nitrogen are the classical index to evaluate kidney function, but with much higher delayed diagnosis and missed diagnosis rate. ${ }^{32}$ Currently, there are still no diagnostic tools for the earlier detection of the SA-AKI development, although some studies have revealed limited predictive values. ${ }^{30} 3133-37$ Recently, a major researchs indicated that renal microvascular abnormalities play a central role in SA-AKI. ${ }^{36-39}$ Disturbances in microcirculatory oxygen delivery may include both decreased fow and diffusion limitation in the setting of organ oedema and inflammation. The microcirculatory derangements such as capillary plugging and microthrombi have been detected in the renal capillaries in animal models of SA-AKI. ${ }^{40}$

Previously, CEUS measurement as a diagnostic tool in monitoring renal microcirculatory perfusion have shown promising results, however, most of them provided information regarding cortical perfusion. ${ }^{41-44}$ Most recently, someone have confirmed that the onset ischaemia tissue in the early stage of SA-AKI was the medulla of the kidney which may change several hours prior to development of oliguria and increased plasma creatinine. ${ }^{45}{ }^{46}$ Medullary hypoxia due to intrarenal blood fow redistribution may be one of the factors causing AKI in sepsis. ${ }^{45}$

Another study, in an experimental model in mice subjected to ischaemia-reperfusion injury demonstrated that CEUS was able to monitor changes in renal microvascular perfusion in space and time. They reported that the outer medullary perfusion decreased disproportionately to the reduction in the cortical and inner medullary perfusion after ischaemia. ${ }^{47}$ The outer medulla appears to be particularly sensitive to development of hypoxia, because of its poor blood supply and the large amount of reabsorptive work performed in the proximal tubules and thick ascending limbs of the loop of Henle. ${ }^{48}$

In line with these studies, our study expected to monitor changes in the renal cortical and medullary microcirculation among septic patients on days 0, 1, 2 and 7 after admission to the EICU. At the same time, we would separate patients into four categories (no SA-AKI, stage 1, stage 2, stage 3 SA-AKI) according to the consensus criteria for sepsis and AKI, and then try to find the correlation between the change of microcirculation in kidney and the development of SA-AKI.

The CEUS for evaluating renal microcirculation has several important advantages, such as being a relatively uncomplicated procedure that can be performed at the patient's bedside during emergency management. Furthermore, a map of the kidney microvasculature can be provided with high temporal and spatial resolution. ${ }^{49}$ In addition, there is no exposure to radiation or risk of nephrotoxicity, which would impair renal perfusion and 
increase the risk of nephrogenic systemic fibrosis. CEUS can be safe for the critically ill patients and even for the paediatric patients. ${ }^{50}$

The present study also has several limitations that will need to be considered. First, the regions of interests of the perfusion map were selected subjectively in the kidney and this might very well represent local microheterogeneities. ${ }^{51}$ To minimise this parameter, three ROIs were drawn for each experimental time point and the results averaged in order to minimise heterogeneity of measurement. Second, this is a prospective observational study conducted in a 10-bed EICU and only 200 patients were to be enrolled in the study; therefore, these available data still remain inconclusive and need to be further validated by multicentre cooperation.

This narrative protocol aims to use CEUS to monitor the renal microcirculation changes in septic patients and attempt to find out the correlation between the changes of microcirculation and the development of SA-AKI.

Contributors All listed authors have participated in the study, and have reviewed and approved the submitted manuscript. NL, ZZ and YH designed this study. JD, LL, $\mathrm{XQ}$ and QJ drafted and submitted the report. $\mathrm{HC}, \mathrm{HZ}$ and $\mathrm{BL}$ revised the report.

Funding The study was supported by the Zhejiang Province Public Welfare Technology Application Research Project (CN) (LGF18H150005) and Scientific research project of Zhejiang Education Commission (Y201737841).

Competing interests None declared.

Patient consent for publication Parental/guardian consent obtained.

Ethics approval The study protocol was approved, as described in the text, on 2 August 2017 by the Ethics Committee of Sir Run Run Shaw Hospital (Zhejiang University Medical College, approval number: 2016C91401).

Provenance and peer review Not commissioned; externally peer reviewed.

Open access This is an open access article distributed in accordance with the Creative Commons Attribution Non Commercial (CC BY-NC 4.0) license, which permits others to distribute, remix, adapt, build upon this work non-commercially, and license their derivative works on different terms, provided the original work is properly cited, appropriate credit is given, any changes made indicated, and the use is non-commercial. See: http://creativecommons.org/licenses/by-nc/4.0/.

\section{REFERENCES}

1. Singer M, Deutschman CS, Seymour CW, et al. The third International consensus definitions for sepsis and septic shock (Sepsis-3). JAMA 2016;315:801-10.

2. Fleischmann C, Scherag A, Adhikari NKJ, et al. Assessment of global incidence and mortality of Hospital-treated sepsis. current estimates and limitations. Am J Respir Crit Care Med 2016;193:259-72.

3. Section 2: AKI definition. Kidney Int Suppl 2012;2:19-36.

4. Uchino S, Kellum JA, Bellomo R, et al. Acute renal failure in critically ill patients: a multinational, multicenter study. JAMA 2005;294:813-8.

5. Bagshaw SM, Lapinsky S, Dial S, et al. Acute kidney injury in septic shock: clinical outcomes and impact of duration of hypotension prior to initiation of antimicrobial therapy. Intensive Care Med 2009;35:871-81.

6. Khwaja A. KDIGO clinical practice guidelines for acute kidney injury. Nephron Clin Pract 2012;120:c179-84.

7. Greenberg JH, Coca S, Parikh CR. Long-Term risk of chronic kidney disease and mortality in children after acute kidney injury: a systematic review. BMC Nephrol 2014;15:184.

8. Odutayo A, Wong CX, Farkouh M, et al. Aki and long-term risk for cardiovascular events and mortality. J Am Soc Nephrol 2017;28:377-87.

9. Gomez H, Ince C, De Backer D, et al. A unified theory of sepsisinduced acute kidney injury: inflammation, microcirculatory dysfunction, bioenergetics, and the tubular cell adaptation to injury. Shock 2014;41:3-11.
10. Post EH, Kellum JA, Bellomo R, et al. Renal perfusion in sepsis: from macro- to microcirculation. Kidney Int 2017;91:45-60.

11. Schrier RW, Wang W. Acute renal failure and sepsis. N Engl J Med 2004;351:159-69.

12. Gómez H, Kellum JA. Sepsis-Induced acute kidney injury. Curr Opin Crit Care 2016;22:546-53.

13. Keir I, Kellum JA. Acute kidney injury in severe sepsis: pathophysiology, diagnosis, and treatment recommendations. J Vet Emerg Crit Care 2015;25:200-9.

14. Fani F, Regolisti G, Delsante M, et al. Recent advances in the pathogenetic mechanisms of sepsis-associated acute kidney injury. J Nephrol 2018;31:351-9.

15. Holthoff JH, Wang Z, Seely KA, et al. Resveratrol improves renal microcirculation, protects the tubular epithelium, and prolongs survival in a mouse model of sepsis-induced acute kidney injury. Kidney Int 2012;81:370-8.

16. Mahoney M, Sorace A, Warram J, et al. Volumetric contrastenhanced ultrasound imaging of renal perfusion. $J$ Ultrasound Med 2014;33:1427-37.

17. Zhang Z, Hong Y, Liu N, et al. Diagnostic accuracy of contrast enhanced ultrasound in patients with blunt abdominal trauma presenting to the emergency department: a systematic review and meta-analysis. Sci Rep 2017;7:4446.

18. Sileanu FE, Murugan R, Lucko N, et al. Aki in low-risk versus high-risk patients in intensive care. Clin J Am Soc Nephrol 2015;10:187-96.

19. Liang KV, Sileanu FE, Clermont G, et al. Modality of RRT and recovery of kidney function after $\mathrm{AKI}$ in patients surviving to hospital discharge. Clin J Am Soc Nephrol 2016;11:30-8.

20. Jung EM, Clevert DA, Schreyer AG, et al. Evaluation of quantitative contrast harmonic imaging to assess malignancy of liver tumors: a prospective controlled two-center study. World J Gastroenterol 2007;13:6356-64.

21. Lichtenstein DA, BLUE-protocol. BLUE-protocol and FALLSprotocol: two applications of lung ultrasound in the critically ill. Chest 2015;147:1659-70.

22. Perera $P$, Mailhot T, Riley D, et al. The rush exam: rapid ultrasound in shock in the evaluation of the critically III. Emerg Med Clin North Am 2010;28:29-56.

23. Dietrich CF, Averkiou MA, Correas J-M, et al. An EFSUMB introduction into dynamic contrast-enhanced ultrasound (DCEUS) for quantification of tumour perfusion. Ultraschall Med 2012;33:344-51.

24. Zhang Z. Univariate description and bivariate statistical inference: the first step Delving into data. Ann Trans/ Med 2016;4.

25. Zhang Z. Model building strategy for logistic regression: purposeful selection. Ann Transl Med 2016;4.

26. Zhang Z, Zhang H, Khanal MK. Development of scoring system for risk stratification in clinical medicine: a step-by-step tutorial. Ann Transl Med 2017;5

27. Hoste EAJ, Bagshaw SM, Bellomo R, et al. Epidemiology of acute kidney injury in critically ill patients: the multinational AKI-EPI study. Intensive Care Med 2015;41:1411-23.

28. Rhodes A, Evans LE, Alhazzani W, et al. Surviving sepsis campaign: international guidelines for management of sepsis and septic shock: 2016. Intensive Care Med 2017;43:304-77.

29. Bradley VE, Shier MR, Lucas CE, et al. Renal hemodynamic response to furosemide in septic and injured patients. Surgery 1976;79:549-54.

30. Kosaka J, Lankadeva YR, May CN, et al. Histopathology of septic acute kidney injury: a systematic review of experimental data. Crit Care Med 2016;44:e897-903.

31. May CN, Calzavacca P, Ishikawa K, et al. Novel targets for sepsisinduced kidney injury: the glomerular arterioles and the sympathetic nervous system. Exp Physiol 2012;97:1168-77.

32. Cheng $X$, Wu B, Liu $Y$, et al. Incidence and diagnosis of acute kidney injury in hospitalized adult patients: a retrospective observational study in a tertiary teaching hospital in Southeast China. BMC Nephrol 2017;18:203.

33. Zhang $\mathrm{Z}$, Lu B, Ni H, et al. Microalbuminuria can predict the development of acute kidney injury in critically ill septic patients. $J$ Nephrol 2013;26:724-30.

34. Zhang Z, Lu B, Sheng X, et al. Cystatin C in prediction of acute kidney injury: a systemic review and meta-analysis. Am J Kidney Dis 2011;58:356-65.

35. Zhang Z, Xu X, Fan H, et al. Higher serum chloride concentrations are associated with acute kidney injury in unselected critically ill patients. BMC Nephrol 2013;14:235.

36. Matejovic M, Ince C, Chawla LS, et al. Renal hemodynamics in AKI: in search of new treatment targets. J Am Soc Nephrol 2016;27:49-58. 
37. De Backer D, Donadello K, Sakr Y, et al. Microcirculatory alterations in patients with severe sepsis: impact of time of assessment and relationship with outcome. Crit Care Med 2013;41:791-9.

38. Dubin A, Pozo MO, Casabella CA, et al. Increasing arterial blood pressure with norepinephrine does not improve microcirculatory blood flow: a prospective study. Critical Care 2009;13.

39. Kanoore Edul VS, Dubin A, Ince C, et al. The microcirculation as a therapeutic target in the treatment of sepsis and shock. Semin Respir Crit Care Med 2011;32:558-68.

40. Lima A, van Rooij T, Ergin B, et al. Dynamic contrast-enhanced ultrasound identifies microcirculatory alterations in sepsis-induced acute kidney injury. Crit Care Med 2018;46:1284-92.

41. Kalantarinia K. Novel imaging techniques in acute kidney injury. Curr Drug Targets 2009;10:1184-9.

42. Schwenger V, Korosoglou G, Hinkel U-P, et al. Real-Time contrastenhanced sonography of renal transplant recipients predicts chronic allograft nephropathy. Am J Transplant 2006;6:609-15.

43. Schneider AG, Hofmann L, Wuerzner G, et al. Renal perfusion evaluation with contrast-enhanced ultrasonography. Nephrol Dial Transplant 2012;27:674-81.

44. Schneider AG, Goodwin MD, Bellomo R. Measurement of kidney perfusion in critically ill patients. Crit Care 2013;17.
45. Calzavacca P, Evans RG, Bailey M, et al. Cortical and medullary tissue perfusion and oxygenation in experimental septic acute kidney injury. Crit Care Med 2015;43:e431-9.

46. Lankadeva YR, Kosaka J, Evans RG, et al. Urinary oxygenation as a surrogate measure of medullary oxygenation during angiotensin II therapy in septic acute kidney injury. Crit Care Med 2018;46:e41-8.

47. Fischer K, Meral FC, Zhang Y, et al. High-Resolution renal perfusion mapping using contrast-enhanced ultrasonography in ischemiareperfusion injury monitors changes in renal microperfusion. Kidney Int 2016:89:1388-98.

48. Evans RG, Ince C, Joles JA, et al. Haemodynamic influences on kidney oxygenation: clinical implications of integrative physiology. Clin Exp Pharmacol Physiol 2013;40:106-22.

49. Harvey CJ, Sidhu PS, Bachmann Nielsen M. Contrast-Enhanced ultrasound in renal transplants: applications and future directions. Ultraschall Med 2013;34:319-21.

50. Rosado E, Riccabona M. Off-Label use of ultrasound contrast agents for intravenous applications in children: analysis of the existing literature. J Ultrasound Med 2016;35:487-96.

51. Rim SJ, Leong-Poi H, Lindner JR, et al. Quantification of cerebral perfusion with "Real-Time" contrast-enhanced ultrasound. Circulation 2001;104:2582-7. 